\title{
AN EMBEDDING THEOREM FOR MATRICES OF COMMUTATIVE CANCELLATIVE SEMIGROUPS
}

\author{
BY
}

\section{JAMES STREILEIN ${ }^{(1)}$}

ABSTRACT. In this paper it is shown that each semigroup which is a matrix of commutative cancellative semigroups has a "quotient semigroup" which is a completely simple semigroup with abelian maximal subgroups. This result is proved by explicitly constructing the quotient semigroup. The paper also gives necessary and sufficient conditions for a semigroup of the type being considered in the paper to be isomorphic to a Rees matrix semigroup over a commutative cancellative semigroup. Several special cases and examples are also briefly discussed.

The study of the semigroups in the title was initiated by Petrich [7] in connection with commutative separative semigroups. It was conjectured in that paper that matrices of commutative cancellative semigroups can be embedded into Rees matrix semigroups over abelian groups. This paper answers the conjecture affirmatively. We also study the embedding and use it to characterize several special cases of matrices of commutative cancellative semigroups.

0 . Preliminaries and summary. We use $S$ to represent a semigroup. If there is a congruence $\rho$ on $r$ for which $S / \rho$ is a rectangular band whose classes are all commutative cancellative semigroups, then we say $S$ is a matrix of commutative cancellative semigroups. Since a rectangular band may be considered as $I \times \Lambda$, the product of a left and right zero semigroup respectively, we will write $S={ }_{I} \bigcup_{\Lambda} S_{i \lambda}$ for a matrix of commutative cancellative semigroups, whose classes are the $S_{i \lambda^{*}}$. In case the rectangular

Received by the editors March 22, 1974.

AMS (MOS) subject classifications (1970). Primary 20M10, $20 \mathrm{M} 30$.

Key words and phrases. Matrix of commutative cancellative semigroups, Rees matrix semigroup, quotient Rees matrix semigroup, quotient group.

(1) This paper contains part of a doctoral dissertation written under the direction of Professor Mario Petrich at Pennsylvania State University. 
band above is just $\Lambda$, a right zero semigroup, we define a right zero union of commutative cancellative semigroups and write $S=\bigcup_{\Lambda} S_{\lambda}$, analogously.

A second concept we will make extensive use of is the Rees matrix semigroup. We denote such a semigroup by $S=\pi(I, G, \Lambda ; P)$, where $I$ and $\Lambda$ are nonempty sets, $G$ is a group, and $P$ maps $\Lambda \times I$ into $G$. The functional value $P(\lambda, i)$ is denoted by $p_{\lambda i}$. Elements of $S$ are of the form $(i, g, \lambda)$ with $i \in I, g \in G, \lambda \in \Lambda$ and multiplication is given by $(i, g, \lambda)(j, h, \mu)=$ $\left(i, g p_{\lambda j} h, \mu\right)$. We call $S$ the Rees matrix semigroup over the group $G$ with sandwich matrix $P$. It is a well-known theorem in semigroup theory that a semigroup is completely simple if and only if it is isomorphic to a Rees matrix semigroup over some group. A completely simple semigroup is a simple semigroup which contains an idempotent $e$ which has the property that if $f$ is another idempotent for which $f=e f=f e$, we must have $e=f$. Any other concepts not defined in the text may be found in Petrich [6] or Clifford and Preston [3].

The main result of $\oint_{1}$ is that matrices of commutative cancellative semigroups are precisely subsemigroups of Rees matrix semigroups over abelian groups. We do this by constructing a special Rees matrix semigroup, called the quotient Rees matrix semigroup, into which a given matrix of commutative cancellative semigroups can be embedded. A characterization of a special type of matrix of commutative cancellative semigroups is given.

$\$ 2$ contains the justification for calling the particular Rees matrix semigroups over abelian groups constructed in $\$ 1$ a quotient Rees matrix semigroup. This is given in a theorem which says that its quotient Rees matrix semigroup is the smallest into which a matrix of commutative cancellative semigroups can be embedded. There are also several other results which give further information about the nature of the embedding.

In $\$ 3$ we use the results already obtained in $\$ \$ 1$ and 2 to characterize Rees matrix semigroups over commutative cancellative semigroups, which generalize the notion of a Rees matrix semigroup over a group. We also consider a restricted family of Rees matrix semigroups over commutative cancellative semigroups.

$\$ 4$ contains a short discussion of several examples. These include free contents, prime quasi-uniserial semigroups and $\pi$-semigroups.

1. The embedding. We start with several definitions which have been used to characterize matrices of commutative cancellative semigroups 
and a lemma which is probably known.

If for any $a, b, c \in S$ we have $a b c=b a c$, then we call $S$ left commutative.

Lemma. If $S=\bigcup_{\Lambda} S_{\lambda}$ is a right zero union of commutative cancellative semigroups, then $S$ is left commutative.

Proof. Let $a, b, c \in S$, so that $a \in S_{\lambda}, b \in S_{\mu}$, and $c \in S_{\eta}$ for some $\lambda, \mu, \eta \in \Lambda$. Since $S_{\eta}$ is a commutative semigroup, we compute $(a b c)(b c)=$ $(b c)(a b c)=b(c)(a b c)=b(a b c) c=(b a)(b c) c=(b a c)(b c)$. Therefore we have $a b c=b a c$, by cancellation in $S_{\eta}$, as required.

We need the following two definitions before we can present our first theorem. We define $S$ to be weakly cancellative if for $a, b, x \in S, a x=b x$ and $x a=x b$ implies $a=b$. A semigroup $S$ is conditionally commutative if for $a, b \in S$ with $a b=b a$, then for any $c \in S$ we have $a c b=b c a$.

Theorem 1. The following conditions on a semigroup $S$ are equivalent:

(i) $S$ is a matrix of commutative cancellative semigroups.

(ii) $S$ is weakly cancellative and conditionally commutative.

(iii) $S$ can be embedded in a Rees matrix semigroup over an abelian group.

Proof. As mentioned in the introduction, Petrich [7] has proved the equivalence of conditions (i) and (ii). Therefore we will start with $S=$ ${ }_{I} \bigcup_{\Lambda} s_{i \lambda}$, a matrix of commutative cancellative semigroups, which is weakly cancellative and conditionally commutative. We will construct a Rees matrix semigroup, $Q_{a}(S)$, over an abelian group into which $S$ can be embedded.

To start the construction, we fix $1 \in I, 1 \in \Lambda$, an element $a \in S_{11}$ and let $G$ be the quotient group over $S_{11}$, written in the natural way as quotients of elements in $S_{11}$. We also define a mapping $P$ from $\Lambda \times I$ into $G$ by

$$
P_{\lambda i}=\frac{a^{2} s t a^{2}}{a s a^{2} t a} \text { for some } s \in \bigcup_{I} s_{j \lambda}, t \in \bigcup_{\Lambda} S_{i \mu} \text {. }
$$

To show that $P$ is single valued we choose another $u \in \bigcup_{I} S_{j \lambda}$ and $v \epsilon$ $\bigcup_{\Lambda} s_{i \mu}$ and will show

$$
\frac{a s t a}{a s a^{2} t a}=\frac{a u v a}{a u a^{2} v a} \text {. }
$$

We obtain the following string of equalities: 


$$
\begin{aligned}
& (\text { asta })(a u a)(a v a)=(\text { asta })(a v a)(a u a)=(a s)\left(t a^{2}\right)\left(v a^{2}\right)(u a) \\
& \left.=(a s)\left(v a^{2}\right)\left(t a^{2}\right)(u a) \quad \text { (by commutativity in } S_{i 1}\right) \\
& =(a s v a)(a t a)(a u a)=(a s v a)(a u a)(a t a)=(a s v a)(a u)\left(a^{2} t a\right) \\
& =(a u)(a s v a)\left(a^{2} t a\right) \quad \text { (by left commutativity in } \bigcup_{\Lambda} s_{1 \mu} \text { ) } \\
& =(a u)(a s) v a^{3} t a=(a s)(a u) v a^{3} t a \quad \text { (by commutativity in } S_{1 \lambda} \text { ) } \\
& =(a s)(a u v a)\left(a^{2} t a\right)=(a u v a)(a s)\left(a^{2} t a\right) \quad \text { (by left commutativity in } \bigcup_{\Lambda} S_{1 \mu} \text { ) } \\
& =(\text { auva })(a s a)(a t a) \text {. }
\end{aligned}
$$

Thus we have established that $P$ is single valued.

Therefore $Q_{a}(S)=\mathbb{M}(I, G, \Lambda ; P)$ is a Rees matrix semigroup over an abelian group. Define a function $\phi_{a}$ on $S$ by:

$$
\phi_{a}(b)=\left(i, a b a / a^{2}, \lambda\right) \text { for } b \in S_{i \lambda} .
$$

It is immediate that $\phi_{a}$ is a function from $S$ into $Q_{a}(S)$. Let $b, c \in S$ with $b \in S_{i \lambda}$ and $c \in S_{j \mu}$. For these elements,

$$
\begin{aligned}
\phi_{a}(b) \phi_{a}(c) & =\left(i, \frac{a b a}{a^{2}}, \lambda\right)\left(j, \frac{a c a}{a^{2}}, \mu\right)=\left(i, \frac{a b a}{a^{2}} p_{\lambda j} \frac{a c a}{a^{2}}, \mu\right) \\
& =\left(i, \frac{a b a}{a^{2}} \cdot \frac{a^{2} b c a^{2}}{(a b a)(a c a)} \cdot \frac{a c a}{a^{2}}, \mu\right)=\left(i, \frac{a b c a}{a^{2}}, \mu\right)=\phi_{a}(b c)
\end{aligned}
$$

using the definition of $p_{\lambda j}$. Hence $\phi_{a}$ is a homomorphism.

Let $b, c \in S$ be such that $\phi_{a}(b)=\phi_{a}(c)$. Then $\left(i, a b a / a^{2}, \lambda\right)=(j$, $\left.a c a / a^{2}, \mu\right)$, implying $i=j, \lambda=\mu$ and $a b a / a^{2}=a c a / a^{2}$. Thus $b, c \in S_{i \lambda}$ and $b c=c b$. This implies $b a c=c a b$ by conditional commutativity. Multiplying by $a$, we have $a b a c=a c a b$ and $b a c a=c a b a$. Using $a b a=a c a$ we obtain $a b a c=a b a b$ and $b a b a=c a b a$ giving $a c=a b$ and $b a=c a$ by cancellation in the respective subsemigroups. These equalities imply $c=b$ by weak cancellation. Thus $\phi_{a}$ is one-to-one and is actually an embedding.

Conversely, it is immediate that a subsemigroup of a Rees matrix semigroup over an abelian group is a matrix of commutative cancellative semigroups.

We call the Rees matrix semigroup $Q_{a}(S)$, constructed in the above theorem, the quotient Rees matrix semigroup for $S$. We note that $p_{\lambda i}$ in the theorem is the identity if $\lambda=1$ or $i=1$. 
We next use Theorem 1 to give a new proof of a part of the following theorem from Petrich [7]. This theorem characterizes medial, weakly cancellative semigroups. A medial semigroup is one which satisfies the identity, $a b c d=a c b d$. We will also have occasion to use square commutativity which means that we always have $(a b)^{2}=a^{2} b^{2}$ for $a, b \in S$. Finally a rectangular abelian group is the direct product of a rectangular band and an abelian group.

Theorem 2. The following conditions on a semigroup $S$ are equivalent:

(i) $S$ is medial and weakly cancellative.

(ii) $S$ is a matrix of cancellative semigroups and is square commutative.

(iii) $S$ is embeddable into a rectangular abelian group.

(iv) $S$ is a subdirect product of a rectangular band and a commutative cancellative semigroup.

Proof. We give only the proof of "(ii) implies (iii)" and refer the reader to Petrich [7] for the remainder. Let $S$ be a matrix of cancellative semigroups, which is also square commutative. Say $S={ }_{I} \bigcup_{\Lambda} S_{i \lambda}$. If $a, b \in S_{i \lambda}$, then $a^{2} b^{2}=(a b)^{2}$. This implies $a b=b a$ by cancellation in $S_{i \lambda}$. Hence $S$ is a matrix of commutative cancellative semigroups. By Theorem 1 we know $S$ can be embedded into the Rees matrix semigroup $Q_{a}(S)$ over an abelian group $G$. Let $(i, a, \lambda),(j, b, \mu) \in S$. By hypothesis $(i, a, \lambda)^{2}(j, b, \mu)^{2}=$ $((i, a, \lambda)(j, b, \mu))^{2}$. Hence $\left(i, a p_{\lambda i} a p_{\lambda j} b p_{\mu j} b, \mu\right)=\left(i, a p_{\lambda j} b p_{\mu i} a p_{\lambda j} b, \mu\right)$, so that $p_{\lambda i} p_{\mu j}=p_{\lambda j} p_{\mu i}$, which implies $p_{\mu j} p_{\lambda j}^{-1} p_{\lambda i}=p_{\mu i}$. This is exactly the requirement given in Petrich [6, IV. 3.3], that a Rees matrix semigroup is the direct product of a rectangular band and a group.

If $x a=x b$ for $a, b, x \in S$ implies $a=b$, then $S$ is left cancellative. Analogously to a rectangular abelian group, a right abelian group is the direct product of a right zero semigroup and an abelian group. These concepts are used in the following corollary of Theorem 2, which is proved in Petrich [7].

Corollary. The following conditions on a semigroup $S$ are equivalent:

(i) $S$ is left commutative and left cancellative.

(ii) $S$ is embeddable into a right abelian group.

(iii) $S$ is a subdirect product of a commutative cancellative semigroup and a right zero semigroup. 
(iv) $S$ is a right zero union of commutative cancellative semigroups.

2. Quotient Rees matrix semigroups. We immediately give the theorem which justifies our earlier definition. As a corollary we will have the earlier known result for right zero unions of commutative cancellative semigroups. We then develop further properties of the embedding $\phi$ constructed in Theorem 1.

Theorem 3. Let $S={ }_{I} U_{\Lambda} S_{i \lambda}$ be a matrix of commutative cancellative semigroups, and $\phi_{a}$ be the embedding of $S$ into $Q_{a}(S)$ given in the proof of Theorem 1. If $\theta$ is a homomorphism of $S$ into $T$, a completely simple semigroup, then there exists $\psi$ a unique homomorphism of $Q_{a}(S)$ into $T$ which makes the following diagram commutative:

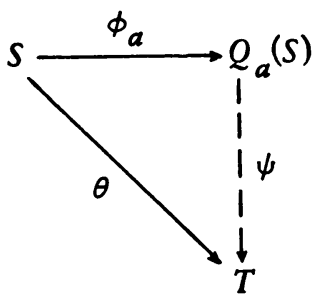

Proof. We will let $S_{11}$ be the subsemigroup of $S$ used to construct $\phi_{a}$ and $Q_{a}(S)=M(I, G, \Lambda ; P)$ as in the proof of Theorem 1 . As we noted after Theorem $1, P$ has all entries in the row and the column containing $P_{11}$ equal to the identity.

By the Rees theorem for completely simple semigroups $T \cong$ $M\left(I^{\prime}, H, \Lambda^{\prime} ; Q\right)$ for some group $H$. Since $\theta$ is a homomorphism it must take elements that commute to elements that commute. Hence $\theta$ induces mappings of $I$ into $I^{\prime}$ and $\Lambda$ into $\Lambda^{\prime}$. We will denote these mappings by primes so that if $b \in S_{i \lambda}$, then $\theta(b)=\left[i^{\prime}, b^{\prime}, \lambda^{\prime}\right] \in T$, where we are using square brackets to distinguish more readily those of $T$ from those of $Q_{a}(S)$. As an additional simplification, we will require that all entries of $Q$ in the row and column containing $q_{1^{\prime} 1^{\prime}}$, be identity elements, which can be done following [3, 3.4].

We next define a mapping $\omega: S_{11} \rightarrow H$ by $\theta(b)=\left[1^{\prime}, \omega(b), 1^{\prime}\right]\left(b \in S_{11}\right)$. Since $S_{11}$ generates its quotient group $G$, we can extend $\omega$ to all of $G$ by $\omega\left(c b^{-1}\right)=\omega(c)(\omega(b))^{-1}$. It is easy to verify that $\omega$ is a homomorphism on $G$.

Using $\omega$, we define the mapping $\psi: M(I, G, \Lambda ; P) \rightarrow M\left(I^{\prime}, H, \Lambda^{\prime} ; Q\right)$ by $\psi(i, b, \lambda)=\left[i^{\prime}, \omega(b), \lambda^{\prime}\right](b \in G)$. We will show that $\psi$ is the required mapping.

Let $(i, b, \lambda),(1, c, \mu) \in Q_{a}(S)$. Then 


$$
\begin{aligned}
\psi((1, b, \lambda)(1, c, \mu)) & =\psi(1, b c, \mu)=\left[1^{\prime}, \omega(b c), \mu^{\prime}\right] \\
& =\left[1^{\prime}, \omega(b) \omega(c), \mu^{\prime}\right]=\left[1^{\prime}, \omega(b) q_{\lambda^{\prime} 1^{\prime}} \omega(c), \mu^{\prime}\right] \\
& =\left[1^{\prime}, \omega(b), \lambda^{\prime}\right]\left[1^{\prime}, \omega(c), \mu^{\prime}\right]=\psi(1, b, \lambda) \psi(1, c, \mu) . .
\end{aligned}
$$

Therefore it is clear that $\psi$ is a homomorphism when restricted to $\bigcup_{\Lambda} S_{i \lambda^{*}}$ Similarly it can be shown that $\psi$ restricted to $\bigcup_{I} S_{i 1}$ is a homomorphism. We also note that it is immediate from the definition of $\omega$ and $\psi$, that we have $\theta(b)=\psi \phi_{q}(b)$ for all $b$ in $S_{11}$, so the diagram commutes on $S_{11}$.

For $b$ in $S_{11}$ and $c$ in $S_{1 \lambda}$, we have $c b$ in $S_{11}$. Thus

$$
\theta(c) \theta(b)=\theta(c b)=\psi \phi_{a}(c b)=\psi \phi_{a}(c) \psi \phi \phi_{a}(b)=\psi \phi_{a}(c) \dot{\theta}(b) .
$$

Since $\theta(c)$ and $\psi \phi(c)$ must be in the same subgroup of $T$, we must have $\theta(c)=\psi \phi(c)$. Hence the diagram commutes on $\bigcup_{I} S_{i 1}$ and similarly it will also commute on $\bigcup_{\Lambda} S_{1 \lambda}$.

We now let $c$ be in $S_{1 \lambda}$ and $d$ be in $S_{i 1}$, so that $\phi(c)=\left(1^{\prime}, c^{\prime}, \lambda^{\prime}\right)$ and $\phi(d)=\left(i^{\prime}, d^{\prime}, 1^{\prime}\right)$ for some $c^{\prime}, d^{\prime}$ in $G$. Therefore

$$
\begin{aligned}
{\left[1^{\prime}, \omega\left(c^{\prime}\right) q_{\lambda^{\prime} i^{\prime}} \omega\left(d^{\prime}\right), 1^{\prime}\right] } & =\left[1^{\prime}, \omega\left(c^{\prime}\right), \lambda^{\prime}\right]\left[i^{\prime}, \omega\left(d^{\prime}\right), 1^{\prime}\right] \\
& =\theta(c) \theta(d)=\theta(c d)=\psi \phi_{a}(c d) \\
& =\psi\left(\left(1, c^{\prime}, \lambda\right)\left(i, d^{\prime}, 1\right)\right)=\psi\left(1, c^{\prime} p_{\lambda i} d^{\prime}, 1\right) \\
& =\left[1^{\prime}, \omega\left(c^{\prime} p_{\lambda i} d^{\prime}\right), 1\right]=\left[1^{\prime}, \omega\left(c^{\prime}\right) \omega\left(p_{\lambda i}\right) \omega\left(d^{\prime}\right), 1^{\prime}\right],
\end{aligned}
$$

which implies that $q_{\lambda^{\prime} i^{\prime}}=\omega\left(p_{\lambda i}\right)$.

We are finally ready to show that $\psi$ is a homomorphism. Let $(i, b, \lambda)$, $(j, c, \mu) \in Q_{a}(S)$. Then

$$
\begin{aligned}
\psi((i, b, \lambda)(j, c, \mu)) & =\psi\left(i, b p_{\lambda_{j}} c, \mu\right) \\
& =\left[i^{\prime}, \omega\left(b p_{\lambda_{j}} c\right), \mu^{\prime}\right]=\left[i^{\prime}, \omega(b) \omega\left(p_{\lambda j}\right) \omega(c), \mu^{\prime}\right] \\
& =\left[1^{\prime}, \omega(b) q_{\lambda^{\prime} j^{\prime}} \omega(c), \mu^{\prime}\right] \\
& =\left[i, \omega(b), \lambda^{\prime}\right]\left[j^{\prime}, \omega(c), \mu^{\prime}\right] .
\end{aligned}
$$

Thus $\psi$ is a homomorphism as required.

We still need to show that the diagram commutes for any $b$ in $S$. We already have this by definition for $b$ in $S_{11}$ and have shown this for all $b$ in $U_{\Lambda} s_{1 \lambda}$ and $U_{I} s_{i 1}$. Therefore we only have to check commutativity for an element $c$ in $S_{i \lambda^{*}}$. We let $b \in S_{11}$, so that $b c b$ is in $S_{11}$. Then $\theta(b, b)=$ 
$\psi \phi_{a}(b c b)$. Hence $\theta(b) \theta(c) \theta(b)=\theta(b c b)=\psi \phi_{a}(b) \psi \phi_{a}(c) \psi \phi_{a}(b)=$ $\theta(b) \psi \phi_{a}(c) \theta(b)$. Since $\theta(c)$ and $\psi \phi_{a}(c)$ are in the same subgroup, it follows that $\theta(c)=\psi \phi(c)$. Thus we have shown that the diagram commutes. It is also clear from the proof that any other map $\psi^{\prime}$ which makes the diagram commutative must take the same action as $\psi$ and thus is the same function and the theorem is proved.

The following corollary for right zero unions of commutative cancellative semigroups is due to Dickinson [4].

Corollary. Let $S=\bigcup_{\Lambda} S_{\lambda}$ be a right zero union of commutative cancellative semigroups, and $\phi$ be the embedding of $S$ into $Q_{a}(S)$ given in Theorem 1. If $\theta$ is an embedding of $S$ into $T$, a right abelian group, then there exists $\psi$, a unique embedding of $Q_{a}(S)$ into $T$ which makes the following diagram commutative:

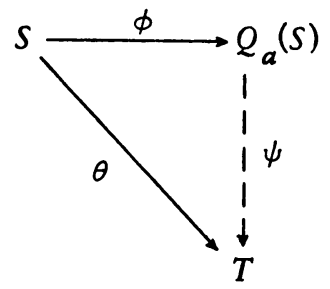

The next proposition shows that quotient Rees matrix semigroups are, up to isomorphism, not dependent upon the choice of the element $a$ used in the construction in Theorem 1.

Theorem 4. Let $S={ }_{I} U_{\Lambda} S_{i \lambda}$ be a matrix of commutative cancellative semigroups, and let $a \in S_{i \lambda}$ and $b \in S_{j \mu}$. If we let $\phi_{a}, \phi_{b}$ be the embeddings of $S$ in $Q_{a}(S), Q_{b}(S)$ as constructed in the proof of Theorem 1 , using $a, b$ respectively, then there exists an isomorphism $\psi$ of $Q_{a}(S)$ onto $Q_{b}(S)$ which makes the following diagram commutative:

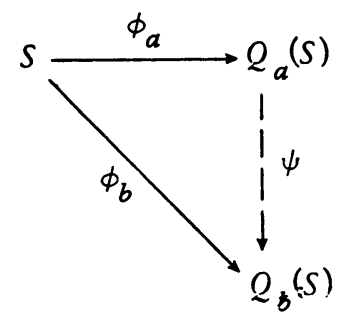

Proof. This follows immediately from Theorem 3. We have unique homomorphisms $\psi_{a, b}: Q_{a}(S) \rightarrow Q_{b}(S)$ and $\psi_{b, a}: Q_{b}(S) \rightarrow Q_{a}(S)$ such that $\psi_{a} \psi_{a, b}=\phi_{b}$ and $\phi_{b} \psi_{b, a}=\phi_{a}$ but then $\phi_{a} \psi_{a, b} \psi_{b, a}=\phi_{a}$. Thus $\psi_{a, b} \psi_{b, a}$ 
is the identity map on $Q_{a}(S)$ and similarly $\psi_{b, a} \psi_{a, b}$ is the identity map on $Q_{b}(S)$. Hence $\psi_{a, b}$ and $\psi_{b, a}$ are inverse isomorphisms.

Corollary. For $S={ }_{l} \bigcup_{\Lambda} S_{i \lambda}$ a matrix of commutative cancellative semigroups, the image of each $S_{i \lambda}$ under any $\phi$ in the proof of Theorem 1 generates the group into which it is embedded.

This is just one of the results needed in the proof of the proposition.

The following corollary could also be derived from the already mentioned work of Dickinson [4].

Corollary. For $S={ }_{I} \bigcup_{\Lambda} S_{i \lambda}$, a matrix of commutative cancellative semigroups, all the $S_{i \lambda}$ have isomorphic quotient groups.

3. Rees compositions. In this section we generalize the construction of Rees matrix semigroups to any semigroup and we characterize those semigroups obtained by using commutative cancellative semigroups in this way. The special case of the direct product of a rectangular band and a commutative cancellative semigroup is also studied.

We need to introduce several standard concepts. A left translation $\lambda$ is a function, written on the left, of $S$ to $S$ which satisfies $\lambda(x y)=\lambda(x) y$ for $x, y \in S$. A right translation $\rho$ is defined similarly when written on the right. A left translation $\lambda$ and a right translation $\rho$ are linked if $x(\lambda y)=$ $(x \rho) y$ for $x, y \in S$. The translation bull of a semigroup, denated by $\Omega(S)$, is the set of pairs of linked left and right translations, $(\lambda, \rho)$, considered as bitranslations. If $(\lambda, \rho),\left(\lambda^{\prime}, \rho^{\prime}\right) \in \Omega(S)$ then multiplication defined by $(\lambda, \rho)\left(\lambda^{\prime}, \rho^{\prime}\right)=\left(\lambda \lambda^{\prime}, \rho \rho^{\prime}\right) \in \Omega(S)$ makes $\Omega(S)$ a semigroup. It is also clear that $(\iota, \iota) \in \Omega(S)$, where $\iota$ is the identity function on $S$ written on the proper side, is the identity for $\Omega(S)$. Hence one can consider the group of units of $\Omega(S)$. A left translation $\lambda$ and a right translation $\rho$ are permutable if $(\lambda x) \rho=$ $\lambda(x \rho)$ for all $x \in S$. A set of bitranslations $T$ is permutable if for any $(\lambda, \rho)$, $\left(\lambda^{\prime}, \rho^{\prime}\right) \in T$, we have that $\lambda$ and $\rho^{\prime}$ are permutable.

We extend the definition of Rees matrix semigroups to $T=\pi(I, S, \Lambda ; P)$, where $I, \Lambda$ are any nonempty sets and $S$ is any semigroup. However $P$ maps $\Lambda \times I$ into a permutable subset of the group of units of $\Omega(S)$. It can be verified that this definition produces a semigroup when $(i, a, \lambda),(j, b, \mu) \in T$ multiply as $(i, a, \lambda)(j, b, \mu)=\left(i, a p_{\lambda j} b, \mu\right)$, where $\left(a p_{\lambda j}\right) b=a\left(p_{\lambda j} b\right)=$ $a p_{\lambda j} b$. We call $M(I, S, \Lambda ; P)$ a Rees matrix semigroup over the semigroup $S$.

Since we are mainly concerned with matrices of commutative cancellative semigroups, we will consider here only Rees matrix semigroups over 
commutative cancellative semigroups. It has been shown by Hall [5] and Dickinson [4] that $\Omega(S)$ for a commutative cancellative semigroup consists of exactly those elements $g$ of the quotient group, say $G$, of $S$ such that $g S \subseteq S$, i.e. the idealizer of $S$ in $G$. It is also immediate that, because of commutativity, all elements $m \in \Omega(S)$ are permutable. Hence in the case of a Rees matrix semigroup over a commutative cancellative semigroup we only require that $p_{\lambda i}$ be a member of the group of units of the idealizer of $S$ in its quotient group. We now present a theorem which characterizes Rees matrix semigroups over commutative cancellative semigroups.

Theorem 5. Let $S={ }_{1} \bigcup_{\Lambda} S_{i \lambda}$ be a matrix of commutative cancellative semigroups. The following statements are equivalent:

(i) For all $a, b \in S$, there exists $c, d \in S$ such that $c b a=b a c, b a=c a$, $d a b=a b d, a b=a d$.

(ii) For all $a \in S$, if $a \in S_{i \lambda}$ then $a S_{j \mu}=a S_{i \mu}$ and $S_{j \mu} a=S_{j \lambda} a$.

(iii) $S$ is isomorphic to a Rees matrix semigroup over any $S_{i \lambda^{*}}$

Proof. (i) implies (ii). Let $a \in S_{i \lambda}$ and $b \in S_{j \mu}$. By the hypothesis of (i) we have an element $d$ such that $d a b=a b d$ and $a b=a d$. Thus $d \in S_{i \mu}$ and we have $a S_{j \mu} \subseteq a S_{i \mu}$. Therefore $b a S_{i \mu} \subseteq b a S_{j \mu}$ since $b a \in S_{j \lambda}$. Since we are in a subsemigroup of a Rees matrix semigroup over a group, we have $a S_{i} \subseteq$ $a S_{j}$ and the first equality in (ii) holds. The second equality in (ii) follows similarly.

(ii) implies (iii). Fix $a \in S_{i \lambda}$ and construct the embedding of Theorem 1. We claim that the image of $S$ in $\pi(I, G, \Lambda ; P)=T$, where $G$ is the quotient group of $S_{i \lambda}$, is a subset of $T$ of the form $I \times S_{i \lambda} \times \Lambda$.

To show this we observe from the proof of Theorem 1 that this is equivalent to showing that $\phi\left(S_{j \mu}\right)=\{j\} \times S_{i \lambda} \times\{\mu\}$ or equivalently $a S_{j \mu} a=$ $a^{2} s_{i \lambda}$. Using the hypothesis in (ii),

$$
\left(a S_{j \mu}\right) a=a\left(S_{i \mu} a\right)=a S_{i \lambda} a=a^{2} S_{i \lambda}
$$

proving that the image is as claimed.

It only remains to show that the $p_{\mu j}$ are in the group of units of the idealizer of $S_{i \lambda}$ in $G$. We already know this if $j=i$ or $\mu=\lambda$ since all such $p_{\mu j}$ are the identity as seen from the proof of Theorem 1. Let $(j, b, \mu)$ and $(k, c, \lambda)$ be in the image of $S$ in $T$. Then $(j, b, \mu)(k, c, \lambda)=(j, d, \lambda)(k, c, \lambda)$ for some $(j, d, \lambda)$ in the image of $S$ in $T$ by (ii). Hence $b p_{\mu k} c=d p_{\lambda k} c=$ $d c$ and thus $b p_{\mu k}=d$. This shows that $p_{\mu k}$ is in the idealizer of $S_{i \lambda}$ in $G$. 
By (ii) for each $d \in S_{i \lambda}$ there exists a $b \in S_{i \lambda}$ such that $(j, b, \mu)(k, c, \lambda)=$ $(j, d, \lambda)(k, c, \lambda)$ which implies $b p_{\mu k}=d$, showing that $p_{\mu k}$ takes $S_{i \lambda}$ onto $S_{i \lambda^{*}}$ Thus $T$ is a Rees matrix semigroup over $S_{i \lambda^{*}}$

(iii) implies (i). Let $S=\pi(I, T, \Lambda ; P)$ be a Rees matrix semigroup over the commutative cancellative semigroup $T$. Then for $a, b \in S$ we have $a=$ $\left(i, a^{\prime}, \lambda\right)$ and $b=\left(j, b^{\prime}, \mu\right)$. It is immediately verified that $c=\left(j, b^{\prime} p_{\mu i} p_{\lambda i}^{-1}, \lambda\right)$ and $d=\left(i, b^{\prime} p_{\lambda j} p_{\lambda i}^{-1}, \mu\right)$ are the elements needed in (i).

Note. Professor Petrich has suggested that the conditions on the sandwich matrix, $P$, can be relaxed in the case of Rees matrix semigroups over commutative cancellative semigroups and he has characterized such semigroups.

We also mention that a result entirely similar to that for direct products of rectangular bands and groups as mentioned in the proof of Theorem 2 can be proved for Rees matrix semigroups over any semigroup. We use this result in the next theorem to characterize direct products of rectangular bands and commutative cancellative semigroups.

Theorem 6. A semigroup $S$ is isomorphic to the direct product of a rectangular band and a commutative cancellative semigroup if and only if $S$ is weakly cancellative, medial and for any $a, b \in S$ there exist $c, d \in S$ for which $b c a^{2}=c a^{2} c$ and $a^{2} d b=d a^{2} d$.

Proof. If $S$ is isomorphic to $T \times B$ where $T$ is a commutative cancellative semigroup and $B$ is a rectangular band, then by Theorem $2, S$ is weakly cancellative and medial. We represent $B$ as $I \times \Lambda$ with $I$ a left and $\Lambda$ a right zero semigroup, respectively. Let $(a,(i, \lambda)),(b,(j, \mu)) \in T \times B$. It is immediately checked that the elements $(b,(j, \lambda))$ and $(b,(i, \mu))$ satisfy the requirements in the statement of this theorem for $c$ and $d$, respectively.

Conversely, assume $S$ is weakly cancellative, medial and satisfies the requirements on elements in the theorem. By Theorem 2, $S$ is a matrix of commutative cancellative semigroups, say $S={ }_{1} \bigcup_{\Lambda} S_{i \lambda^{*}}$ Let $a, b \in S$ so that by hypothesis there exist $c, d \in S$ with $b c a^{2}=c a^{2} c$ and $a^{2} d b=d a^{2} d$. If $a \in S_{i \lambda}, b \in S_{j \mu}$ then $c \in S_{j \lambda}$ and $d \in S_{i \mu}$. We have $b c a^{2}=b a c a$ by mediality and $a c a=a^{2} c$ by right commutativity in $\bigcup_{I} S_{k \lambda}$ from the corollary after Theorem 2. Thus $b a^{2} c=c a^{2} c$ and since by Theorem 1 we are in a subsemigroup of a Rees matrix semigroup over an abelian group we have $b a=c a$. Hence by Theorem $5, S$ is a Rees matrix semigroup over any of the commutative cancellative semigroups $S_{i \lambda}$. 
We now let $(i, a, \lambda),(j, b, \mu),(k, c, \mu)$ and $(l, d, \gamma) \in S$. By mediality $(i, a, \lambda)(j, b, \mu)(k, c, \mu)(l, d, \gamma)=(i, a, \lambda)(k, c, \mu)(j, b, \mu)(l, d, \gamma)$. This implies that $p_{\lambda j} p_{\mu k} p_{\mu l}=p_{\lambda k} p_{\mu j} p_{\mu l}$, or $p_{\lambda j} p_{\mu j}^{-1} p_{\mu k}=p_{\lambda k}$ using commutativity and cancellation in the quotient group of the $S_{i \lambda}$ for which we do the embedding in Theorem 1. Hence, by the result referred to in Theorem 2, $S$ is actually isomorphic to the direct product of the commutative cancellative semigroup $S_{i \lambda}$ and the rectangular band $I \times \Lambda$.

Corollary. For a semigroup $S$ the following are equivalent:

(i) $S$ is a Rees matrix semigroup of commutative, cancellative semigroups with $|I|=1$.

(ii) $S$ is a left commutative, left cancellative semigroup and for $a, b \in S$ there exists a $c \in S$ such that baca $=c a^{2} c$.

(iii) $S$ is left commutative, left cancellative and $S a \subseteq$ aS for all a $\in S$.

(iv) $S$ is isomorphic to the direct product of a commutative, cancellative semigroup and a right zero semigroup.

The equivalence of (i), (iii) and (iv) can be found in Petrich [7].

4. Examples. We discuss briefly free contents, prime quasi-uniserial semigroups and Rees matrix semigroups over r-semigroups.

As defined by Tamura [10], the free content on two generators, denoted by $C(a, b)$, is the subsemigroup of $F(a, b)$, the free semigroup on the two generators $a$ and $b$, which consists of all words that contain both $a$ and $b$ at least once.

It has been shown by Shafer [8] that any countable semigroup can be embedded in $C(a, b)$.

Shafer [8] denotes by $\lambda$ the congruence on $F(a, b)$ generated by the identities $a=a^{2}$ and $b=b^{2}$. He has shown that $C(a, b) / \lambda$ is a matrix of infinite cyclic semigroups.

As a second example we mention prime quasi-uniserial semigroups as defined by Behrens [1], [2]. Let $I$ be any set, $G$ be the infinite cyclic group generated by $\omega, C$ be the subsemigroup of $G$ consisting of $\left\{\omega^{s} \mid s=0,1, \ldots\right\}$, and $\pi$ be a function from $I \times I$ to the nonnegative integers, $\{0,1,2, \ldots\}$, which satisfies the following conditions. If we denote $\pi(i, j)$ by $(i j), \pi$ must satisfy:

1. $(i i)=0$,

2. $(i j)+(j k) \geq(i k)$,

3. $(k j)+(j i)>0, i \neq j$. 
The set $S=I \times G \times I$ is a prime quasi-uniserial semigroup when we define multiplication by $\left(b, \omega^{s}, i\right)\left(j, \omega^{t}, k\right)=\left(b, \omega^{s+t+(i j)}, k\right)$. It is easy to see that $S$ is a matrix of commutative cancellative semigroups. The conditions on $\pi$ are also equivalent to the conditions that all $e_{i}=\left(i, \omega^{0}, i\right), i \in I$, are idempotent and that $T=\bigcup_{i j} C e_{i} e_{j}$ is a subsemigroup of $S$ containing no further idempotents, where $\omega^{s}\left(h, \omega^{t}, i\right)=\left(h, \omega^{t+s}, i\right)$. It is easily checked that $T$ is a matrix of commutative cancellative semigroups. Behrens uses such semigroups in the study of prime, arithmetic rings with identity.

In fact both of the above examples can be considered as the more restrictive case of matrices of $\gamma_{\text {-semigroups. An }}$-semigroup is an archimedean commutative cancellative semigroup without idempotents. Tamura [9] has constructed all $\Re$-semigroups as pairs $(G, I)$, where $G$ is an abelian group and $I$ maps $G \times G$ into $N$, the nonnegative integers, and satisfies the following conditions:

(i) $I(\alpha, \beta)+I(\alpha \beta, \gamma)=I(\alpha, \beta \gamma)+I(\beta, \gamma)(\alpha, \beta, \gamma \in G)$,

(ii) $I(\alpha, \beta)=I(\beta, \alpha)(\alpha, \beta \in G)$,

(iii) $I(\epsilon, \epsilon)=1$ where $\epsilon$ is the identity of $G$,

(iv) for each $\alpha \in G$ there exists $m>0$ such that $I\left(\alpha^{m}, \alpha\right)>0$. The multiplication on $S=N \times G$ defined by $(m, \alpha)(n, \beta)=(m+n+I(\alpha, \beta)$, $\alpha \beta)$ makes $S$ an $\pi$-semigroup.

Hall [s] has characterized the group of units of the idealizer of $S$ in its quotient group, which we denote by $\Sigma(S)$. The characterization is that

$$
\Sigma(S)=\left\{[0, g] \mid g \in G, I(g, h)>0 \text {, and } I\left(g^{-1}, h\right)>0\right.
$$

for all $\left.h \in G, I\left(g, g^{-1}\right)=1\right\}$,

where $[0, g]$ is a function on $S$ defined by $[0, g](n, h)=(n+I(g, b)-1, g h)$ using the Tamura representation $(G, I)$ given above.

If $G$ is any abelian group then $I: G \times G \rightarrow\{1\}$ satisfies the above four conditions. For $S=N \times G, \Sigma(S)=\{[0, g] \mid g \in G\}$. We can use these facts to construct many Rees matrix semigroups over the $\pi$-semigroup $S$.

As another less trivial example, let $G=\left\{e, a, a^{2}, a^{3}\right\}$ be the cyclic group of order 4. If we define $I$ by $I\left(e, a^{i}\right)=1$ for $i=1,2,3,4, I(a, a)=0$, $I\left(a, a^{2}\right)=1, I\left(a, a^{3}\right)=2, I\left(a^{2}, a^{2}\right)=3, I\left(a^{3}, a^{2}\right)=3$ and $I\left(a^{3}, a^{3}\right)=2$, then $I$ satisfies the above conditions and $\Sigma(S)=\left\{[0, e],\left[0, a^{2}\right]\right\}$.

\section{REFERENCES}

1. E. Behrens, Ring theory, Academic Press, New York, 1972. 
2. E. Behrens, The arithmetic of the quasi-uniserial semigroups without zero, Canad. J. Math. 23 (1971), 507-516. . MR 44 \#2677.

3. A. H. Clifford and G. B. Preston, The algebraic theory of semigroups. Vol. I, Math. Surveys, no. 7, Amer. Math. Soc., Providence, R. I., 1961. MR 24 \#A2627.

4. R. P. Dickinson, Jro, On right-zero unions of commutative semigroups, Pacific J. Math. 41 (1972), 355-364. MR 46 \#5488.

5. R. E. Hall, The translational hull of an $\pi$-semigroup, Pacific J. Math. 41 (1972), 379-389. MR 46 \#5495.

6. M. Petrich, Introduction to semigroups, Merrill, Columbus, Ohio, 1973.

7. - Normal bands of commutative cancellative semigroups, Duke Math. J. 40 (1973), 17-32. MR 47 \#381. lished)。

8. J. Shafer, Homomorphisms and subdirect products of free contents (unpub-

9. T. Tamura, Commutative nonpotent archimedian semigroup with cancellation law. I, J. Gakugei Tokushima Univ. 8 (1957), 5-11. MR 20 \#3224.

10. - The study of closets and free contents related to the semi-lattice decomposition of semigroups, Semigroups (Proc. Sympos., Wayne State Univ., Detroit, Mich.,. 1968), Academic Press, New York, 1969, Pp. 221-260. MR 46 \#5504.

DEPARTMENT OF MATHEMATICS, PENNSYLVANIA STATE UNIVERSITY, UNIVERSITY PARK, PENNSYLVANIA 16802

THE ARMY MATERIAL SYSTEMS ANALYSIS AGENCY, ABERDEEN PROVING GROUNDS, ABERDEEN, MARYLAND 21005

Current address: R.D. 5 Box 218, Elkton, Maryland 21921 